\title{
Algorithm development and hardware implementation for medical image compression system: a review
}

\author{
Noor Huda Ja'afar ${ }^{1}$, Afandi Ahmad ${ }^{2}$ \\ ${ }^{1}$ Universiti Kuala Lumpur Malaysian Institute of Industrial Technology, Bandar Seri Alam, Malaysia \\ ${ }^{2}$ Department of Computer Engineering, Faculty of Electrical and Electronic Engineering, \\ Universiti Tun Hussein Onn Malaysia (UTHM), Malaysia
}

\begin{tabular}{l} 
Article Info \\
\hline Article history: \\
Received Sep 2, 2019 \\
Revised Dec 3, 2019 \\
Accepted Dec 17, 2019 \\
\hline
\end{tabular}

\section{Keywords:}

Algorithm

Hardware

Medical image compression

Software

\begin{abstract}
In the high-tech world, medical imaging is very important to diagnose and analyze illness inside human body. The increasing number of patients annually has continuously growth the amount of medical imaging data generated and directly causes a demand for data storage. Generally, medical images are rich with data, where these data are important for diagnosing purpose. However, some of the data represents redundant information and sometimes can be discarded. Thus, the research area on medical image compression dealing with three-dimensional (3-D) modalities need to be given more attention and exploration. The algorithm development using wavelet transform with software implementation are the famous topics explored among researchers, whilst fewer works have been done in utilizing curvelet transform in medical image compression. Along with that, very limited hardware implementation of 3-D medical image compression is discovered. In term of performance evaluation, most of the previous works conducted objective test compared with subjective test. To fill in this gap, medical image compression system will be reviewed, with the aim to identify the recent method used in medical image compression system. This paper thoroughly scrutinizes the recent advances in medical image compression mainly in terms of compression method, algorithm development with software and hardware implementations and performance evaluation. In conclusion, the overall picture of the medical image compression landscape, where most of the researchers more focused on algorithm development or software implementations without having the combination of software and hardware implementations.
\end{abstract}

Copyright (C) 2020 Institute of Advanced Engineering and Science. All rights reserved.

\section{INTRODUCTION}

In the high-tech world, various types of medical imaging modalities such as computed tomography (CT), magnetic resonance imaging (MRI), positron emission tomography (PET), ultrasound (US) and X-ray have been used to diagnose and analyze illness inside human body. Generally, the size of medical images is larger compared to the standard images. This is due to the fact that medical images are rich with data, where these data are important for diagnosing purpose. Moreover, the increasing number of patients annually has continuously growth the amount of medical imaging data generated and directly causes a demand for data storage [1]. These issues have provided an impulse to the development of telemedicine and teleradiology applications [2-4]. In these fields, medical image compression is important to reduce the size of the data generated without degrading the quality of the image [5]. As a rule, image compression aims at reducing the number of bits needed to represent an image by removing the spatial and spectral redundancies as much as 
possible. Furthermore, many current compression schemes provide a very high compression rate but with considerable loss of quality. But in the case of medical field there is a need to achieve no deterioration in image quality.

The fundamental actions of the algorithms used in medical image processing applications involve matrix operations, mostly matrix transforms include Fast Fourier Transform (FFT), Discrete Wavelet Transform (DWT), some recently developed transforms such as finite Radon, curvelet and ridgelet transform [6-7]. Therefore, there is a real need for high-performance systems, whilst keeping architectures flexible to allow for quick upgradeability with real-time applications [2].

From the hardware perspective, there are several possible devices, such as digital signal processors (DSPs), application specific integrated circuits (ASICs), multiple processors system platform and field programmable gate arrays (FPGAs). Eventhough DSPs are used in a wide range of applications, but the instruction sets are often highly irregular and this processor is inefficient regarding performance and power consumption [8]. Meanwhile, ASICs architecture is easier and faster to design since it only need fewer layers to fabricate and it contain fixed arrangement of integrated circuit for a particular use. Unfortunately, the obvious disadvantage of ASICs is no programmable resources are provided which not allow them to be reconstructed [9].

Furthermore, reconfigurable hardware in form of FPGAs appears as viable system building block in the construction of high-performance systems. The capability to develop a programmable circuit architecture with the flexibility of computational, memory, speed and power requirement, FPGAs seem an ideal candidate to be proposed as a hardware technology for prototype a simple, moderate and complex applications [2], [10]. Indeed, other advantages offered by FPGAs are massive parallelism capabilities, multimillion gate counts and special low-power packages $[6,11]$.

A close examination of the algorithms used in real-time medical image processing applications has revealed that it involves many fundamental actions such as matrix or vector operations and a repeated complex computation [1,5]. The repetitive medical image algorithms can be computed using two implementation strategies; software and hardware. Both methods appear with different regulation. To carry out the software implementation, a set of instructions are built up using any software available in the market such as LabVIEW, C-program or MATLAB. Later, these instructions will be implemented into a suitable device to execute the computations. Meanwhile, hardware implementation used a specific task for certain applications and it provides highly optimized resources. In fact, to achieve high performance solutions for image and signal processing, marrying the benefits of both software and hardware implementation is truly the right way [12].

Recently, the compression algorithms are being developed with various types of techniques such as pipelining, distributed arithmetic (DA) and systolic array (SA). Additionally, having a hardware implementation that is solely dedicated to the compression algorithms will give tremendous results [2, 13]. Despite its complexity, there has been an interest in three-dimensional (3-D) curvelet transform implementation on various platforms. Previous surveys have classified this research field into two categories: algorithms development [14-16] and algorithms with hardware implementation [11, 12]. Moreover, a huge gap stills remain for further research in exploiting reconfigurable computing for 3-D medical image compression in the form of two major limitations:

a) Discrete curvelet transform algorithm has not been widely explored in designing 3-D medical image compression system. However, various types of DWT filters such as Haar, Symlet and Daubechies are extensively used in several implementations [14, 17, 18].

b) Image compression is a well-established research area. However, medical image compression especially dealing with 3-D modalities are still considered as pre-mature research area. Although newer compression algorithm optimizations have been proposed, a very limited hardware implementation of 3-D medical image compression is discussed [19, 20].

Generally, studies from which this paper was prepared were basically derived from different databases. Out of 17 studies gleaned 8 on compression algorithm development and 9 were on medical image compression system. The review classification is done according to transform algorithm, evaluation parameters, types of compression, types of implementation and evaluation systems. The summary of compression algorithm developments and the summary of medical image compression system are recorded in Table 1 and Table 2 respectively and the reviewed details are explained in the next section.

The rest of the paper is organized as follows. An overview of the classification of compression method is summarized in Section 2. Section 3 discuss the algorithm developments for medical image compression, whilst the compression algorithms with hardware implementations are explained in Section 4. Finally, a concluding remark are given in Section 5. 


\section{CLASSIFICATION OF COMPRESSION METHODS}

A basic operation of image compression system involves three processes, which are transformation, quantization and entropy coding as shown in Figure 1. An array of input pixels will go through the first transformation process for having the transformed coefficients as the output. Then, the transformed coefficients are quantized to produce a finite number of levels. In addition, the entropy coding process is applied to the finite set of numbers to give an additional compression.

Comparing the three processes involved in the image compression system operation, the transformation process is the main important process than the others [5]. This is due to the fact that an array input pixel needs to be processed by the transformation block first, before the transformed coefficients are applied to the next process. In technical view, an image is containing of smooth and sharp variations. The smooth variation is the base of the image and represents the low-pass variation. On the other side, the sharp variation represents the high-pass variation and both variations are added together to gives a detailed image. In this context, the purpose of the transformation process is to separate the smooth and sharp variations.

As the features of medical image processing involves many fundamental actions such as matrix or vector operations, thus a variety of algorithms has been developed for compressing medical images. Previous surveys have classified image transform coding algorithms into two methods: using wavelet transform [21], $[14,17]$ and curvelet transform $[22,23]$. A wavelet is a mathematical tool to extract information from different kinds of data such as audio signals and images [24]. Meanwhile, curvelet transform introduced in the year 2000 [25], holds potential in addressing the shortcomings of wavelets. Both image coding algorithms have their own advantages in analyzing the image and signal processing.

An implementation of 3-D Haar Wavelet Transform (HWT) using Distributed Arithmetic (DA) technique for medical image compression system is presented in [13]. This paper is only concern on the transformation process based on Discrete Wavelet Transform (DWT) algorithm with aim to reduce the size of medical image and to speed up the processing of large medical volumes. The advantages offered by DWT which is a multiresolution analysis allows the architectures to be designed with cascade method. In other words, the 3-D HWT is split into three 1-D HWT with transpose module in between. The first 1-D HWT is applied along the rows of the matrix followed by another 1-D HWT along the columns of the transformed matrix. While the third 1-D HWT is applied to the corresponding pixels in each $N$ sub-images.

In addition, this paper explores the benefits of using DA technique in design the proposed architectures. This design technique computes an algorithm that performs a multiplication operation using Look Up Table (LUT) instead of logic elements. In addition, the main advantage offered by the DA design technique is all the pre-computing possible products can be stored in LUTs, thus reduce the area implementation. Figure 2 illustrates the proposed architecture for 1-D HWT. The LUT contains eight possible values that have been computed from the HWT coefficients. To access the LUT content, two vector $X_{l}$ and $X_{2}$ are used with sign bit signal, $T_{s}$.

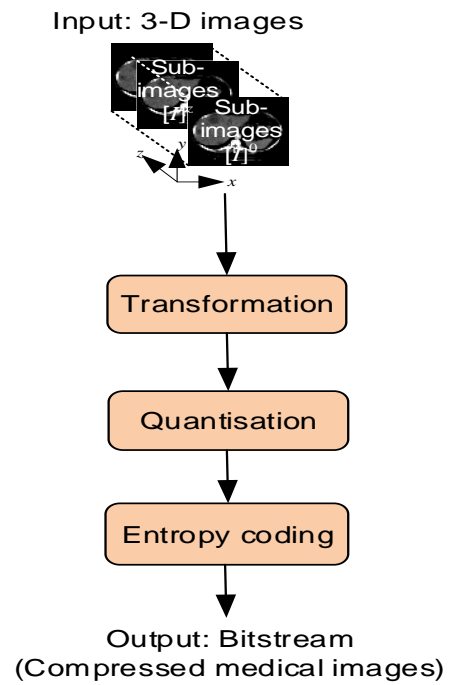

Figure 1. Block diagram for image compression system

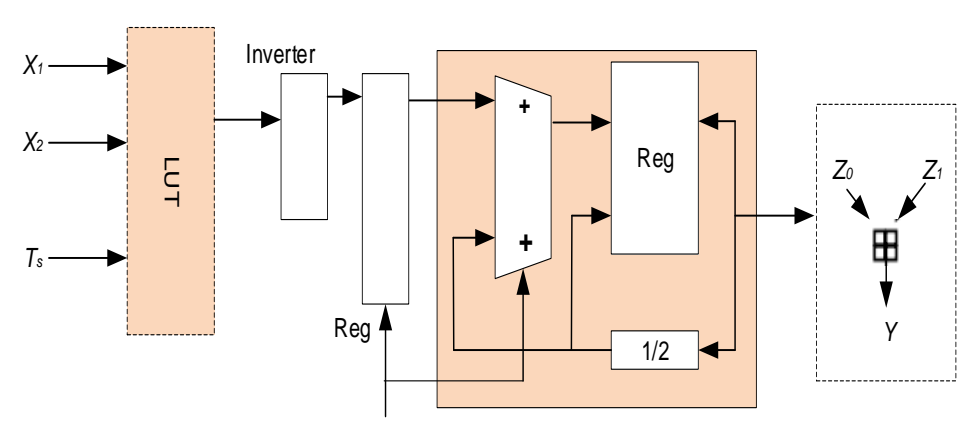

Figure 2. Proposed architecture for 1-D HWT [13] 
To evaluate the compression system, magnetic resonance imaging (MRI) and computer tomography (CT) medical image modalities are used and the proposed architectures are implemented on SubRIO-9632 board National Instrument. The results briefly show that the implementation of 3-D HWT using DA design technique provides saving area and power consumption. Another issue on the medical image compression is presented by D. Ravichandran et. al. [26]. A new medical image compression coding algorithm using wavelet transform is proposed. The difficulty in transmitting and receiving a large volume of medical image with relatively limited bandwidth have become a reason to implement the image compression system application. The proposed compression coding is based on DWT. The availability offered by DWT gives advantages to the architecture design, in which the level of the decomposition can be set into a higher level.

As shown in Figure 3, the coding section includes forward DWT to generates the wavelet coefficients. Then, a hard thresholding is applied to quantize the coefficient vectors and finally the image is compress using Huffman variable encoding technique. A similar concept is applied in the decoding section to reconstruct the compressed image. However, an inverse DWT is used to regenerates the image pixels.

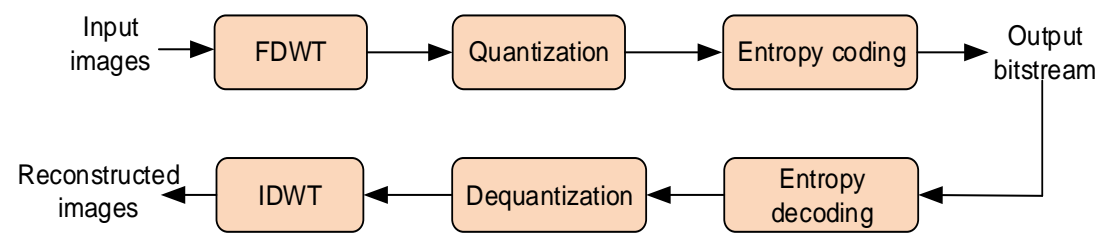

Figure 3. Compression system using DWT [26]

In this work, a various wavelet family are used along with the DWT to analyze the most appropriate wavelets for compressing medical image. Furthermore, the selected wavelet filters are Haar, Daubechies, Coiflet, Symlet and Biorthogonal. To evaluate the compression performance, a large number of test images are used such as X-ray, MRI, CT and ultrasound (US). Results from the various test images have shown that Biorthogonal wavelet performs better than Haar wavelet in terms of peak signal-to-noise ratio (PSNR).

The implementation of medical image compression technique based on hybrid DWT with neural network was presented in [27]. Back propagation neural network (BPNN) with DWT algorithms are used to get better compressed image without degrading the quality of the image. The main advantages offered by this hybrid method are it provides very less compression ratio and high PSNR values. The proposed new compression method is illustrated as a block diagram in Figure 4.

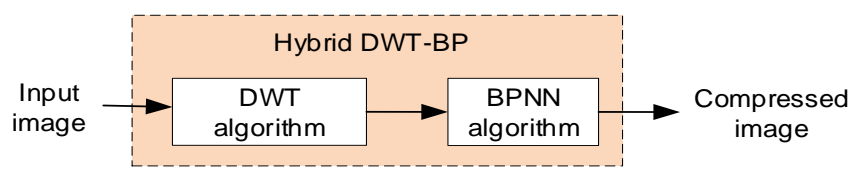

Figure 4. Block diagram of hybrid DWT-BP [27]

In wavelet transform, the decomposition of an image consists of approximation and detailed of images, whilst the back-propagation algorithm requires three layers which are input, hidden and output layers to performs the compression image. From the result, hybrid both the combinations of DWT and BPNN algorithms provides better performance in terms of PSNR compared to implement the algorithm individually.

Most of the proposed compression system use wavelet transform method since it provides multiresolution analysis and gives better results compared to DCT. However, recent works have shown that wavelet transform fails along singularities along edges in multiple directions. Thus, a new compression scheme based on curvelet transform method was introduced in [28]. The first generation of curvelet transform was developed by Candes and Donoho in 1999 and it was a drawback from the wavelet transforms algorithm. Moreover, the main purpose is to represent edges and curves better than wavelet transform. Figure 5 illustrates the decomposition of curvelet transform.

Interestingly, the curvelet and wavelet sub-bands have a relationship since curvelet is an extension of wavelet. Thus, the curvelet sub-band decomposition is similar like wavelet in which the input image is divided into resolution layers. Each layer contains details of different frequencies which are low-pass filter and high- 
pass filter. Another step involves in the curvelet decomposition are smooth partitioning, renormalization and ridgelet analysis. Even though the result shows a good performance in terms of PSNR and compression ratio (CR), but the researcher has identify that the ridgelet tranform give a slow effect to the compression scheme performance. Consequently, the next curvelet generation it will eliminate the presence of the ridgelet transform.

Another image compression scheme dealing with curvelet transform was reported in [29]. In order to overcome the limitation of wavelet transform on image edges and the slow performance of ridgelet transform, the second generation of curvelet transform which called as fast discrete curvelet transform (FDCT) is implemented in image compression system. Moreover, curvelet transform algorithm is suitable to be used with medical image since it dealing with curvy portions. In general, there are two methods to generate the curvelet coefficients, using wrapping or unequal space fast fourier transform (USFFT) methods. The wrapping method has better and faster computation time and thus has been selected in this work. The algorithmic approach for the proposed image compression system is depicted in Figure 6.
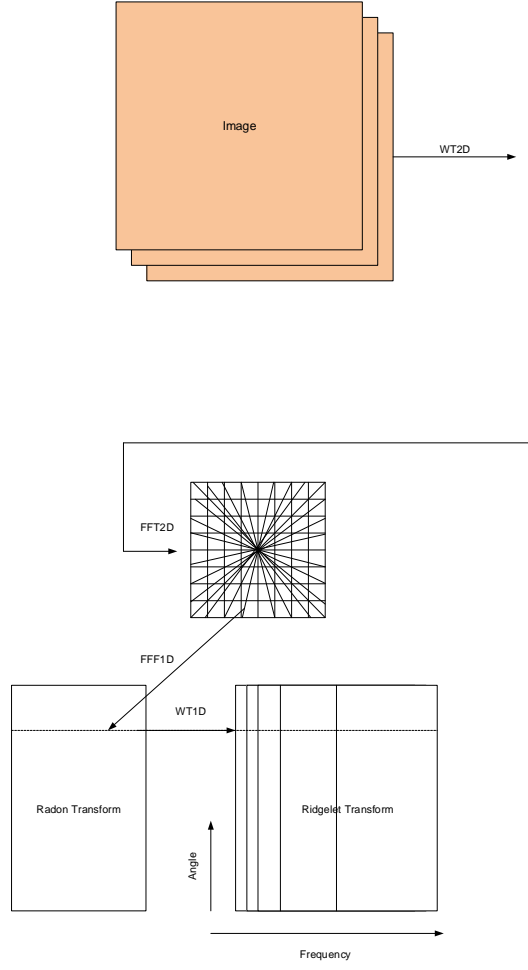

Figure 5. Curvelet transform decomposition [28]
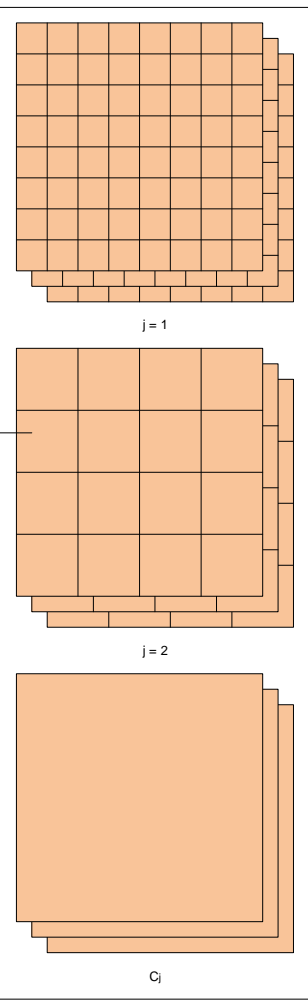

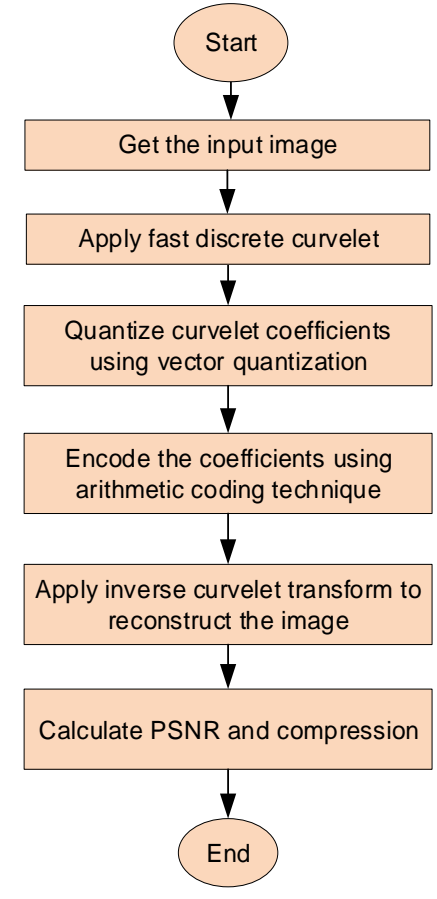

Figure 6. Algorithmic approach for image compression [29]

In the transformation block, the input pixels are processed using FDCT algorithm with wrapping method. Then, the curvelet coefficients generated from the FDCT process are quantized using vector quantization before being encoded using arithmetic coding technique. In addition, the inverse curvelet transform algorithm is applied to reconstruct the compressed image. The proposed compression scheme has been tested using various medical images such as MRI, CT and ultrasound. Moreover, the most parameters used to evaluate the compression performance are PSNR and CR. From the results, it proves that curvelet transform method provides significant improvement in PSNR and CR compared to DCT and wavelet transform.

A variety of method and approach were conducting to achieve better results for image compression. Wavelet transform-based method is more popular among researcher due to the mutliresolution analysis and improve the compression ratio. However, the recent works have discovered the disadvantage of using wavelet transform in which it has an image edges limitation. Moreover, another weakness of wavelet transform is it requires $\frac{1}{N}$ squared error to represent an edge. Thus, curvelet transform-based method is start to be explored in image compression applications. On the other side of image compression, most of the researchers were focussing on objective performance evaluations such as PSNR, MSE and CR. The objective test successfully 
achieved the proposed algorithm and architecture. Furthermore, future potential research would explore more on curvelet transform method with different design techniques such as distributed arithmetic (DA) and systolic array (SA).

\section{ALGORITHM DEVELOPMENT FOR MEDICAL IMAGE COMPRESSION}

In general, compressing medical image is more challenging compared to non-medical image. This is because for medical images, the compression algorithms are complex and it should always be stored in lossless format even though sometimes lossy format is acceptable. Thus, a key aspect of medical image compression was the algorithm or architecture development. The algorithm can be developed using various programming medium such as LabVIEW, MATLAB, Python and Verilog. The performances of the algorithm development in a simulation mode were evaluated.

One of the proposed algorithms for medical image compression system was presented by [15]. A wavelet-based medical image compression using sparsification and coding was proposed. The DWT decomposition were used to separates the sub-bands of the image pixels as shown in Figure 7(a). A framebased method is applied in which the image was separated into many tiles and a larger tile size can avoid the blocking artifacts. Moreover, the outputs giving the detail coefficient, $H$ and approximation coefficient, $L$ from the high-pass and low-pass filter respectively.

Different types of wavelet filter such as Biorthogonal, Coiflet, Daubechies and Symlet have been chosen to get a performance comparison. Furthermore, to achieve a better compression ratio, algorithm based on adaptive scan wavelet difference reduction (ASWDR) is selected. As depicted in Figure 7(b), after the DWT decomposition process, the sub-bands or wavelet coefficients are arranged in linear path with parent and successive nodes. An adaptive linear path scanning, $(x[1], x[2], x[3], \ldots, x[n-1], x[n])$ is chosen for refinement pass with different threshold iteration. The results show that by using Biorthogonal wavelet it provides better performance in terms of compression ratio. Moreover, the selected wavelet filter also prove that it is suitable to be used for medical image compared to others wavelet filters and $97.5 \%-99.5 \%$ of structure similarity is obtained.

Another algorithm development for medical image compression system was reported in [30]. A combination of DWT and DCT methods were used to develop the proposed novel algorithm. As shown in Figure 8, an input images were decomposed into different sub-bands using DWT. Because of DWT method allows the decomposition process to be done into different levels, thus the wavelet coefficients can be manipulated with another algorithm such as DCT. In addition, the wavelet coefficients were separated into non-overlapped $8 \times 8$ blocks for last wavelet pass. The $H L, L H$ coefficients are applied with DCT, whilst the $H H, L L$ are quantized with DWT.

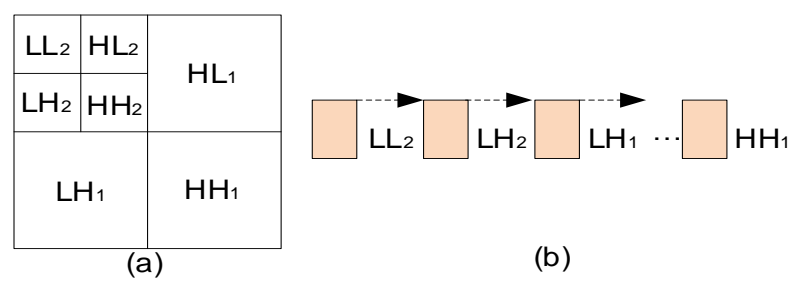

Figure 7(a). Image decomposition using ASWDR algorithm (b). Linear order and scanning path of sub-bands $[15$

In order to test the proposed algorithm, three medical images the size of $512 * 512$ pixels (X-ray), $256 * 256$ pixels (MRI) and 512*512 pixels (pulmonary) have been used. The performance is evaluated by three criteria which are CR, subjective quality and PSNR. The experiment results have shown that the proposed algorithm provides higher compression ratio and avoid blocking artifacts compared to conventional method (JPEG). Furthermore, the proposed algorithm also allows good localization both in spatial and frequency domain (DWT-DCT).

Lossless compression of medical images for better diagnosis is described in [31]. In this work, a region of interest (ROI) that represent the important information of the image and non-region of interest (nonROI) that represent the background of the image are highlighted. Interestingly, the DWT algorithm is used to achieve the lossless compression for the ROI image. Whilst, an embedded zerotree wavelet (EZW) is apply to 
the non-ROI image to get the lossy compression. Figure 9 illustrates the proposed lossless and lossy compression system.

The image is segmented into ROI and non-ROI parts before being processed into a different compression scheme. The main information data for the image was compressed using DWT method with selected Haar and Daubechies wavelete filters. On the other side, the background of the image was compressed using EZW method. Results have shown that for the comparison between different wavelet filters, the Daubechies has significantly improved the MSE and PSNR values. However, the proposed compression scheme has to manually select the ROI image using free hand tool available in MATLAB.

Another medical image compression scheme using EZW method was presented in [1]. A parent child relationship in a zerotree structure allows the bits stream to be created together in order of importance. As shown in Figure 10, the EZW scanning order is based on zig-zag sequence. Using zig-zag method, a large amount of zeros coefficients can be confederated. Thus, a higher compression ratio can be achieved. In addition, a successive-approximation technique and adaptive arithmetic are applied for quantization and bit generation respectively. To test the proposed compression scheme, the medical test images of MRI, Ultrasound and X-ray was downloaded from the online free medical database. From the simulation results, it reveals that the PSNR values are reliant on the type of image and its storage format used. Moreover, the quality of the reconstructed images not only depends on the wavelet-based image compression, but also depends on the image characteristics and attributes.

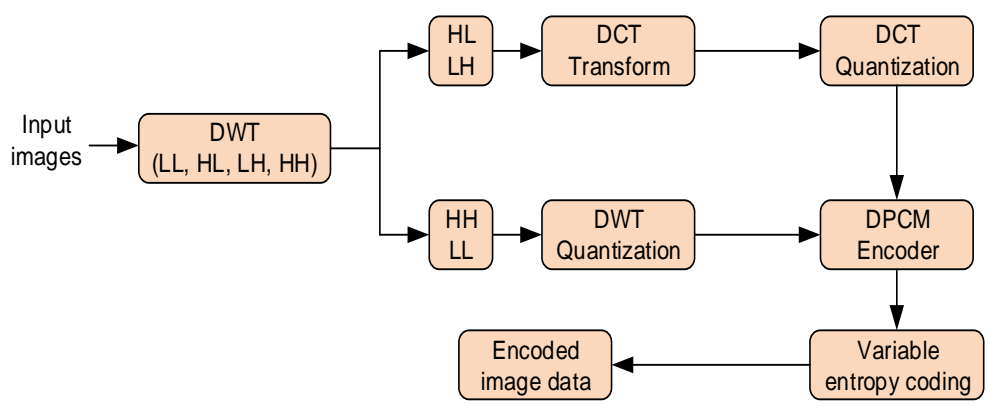

Figure 8. Propose novel algorithm using DWT and DCT [30]

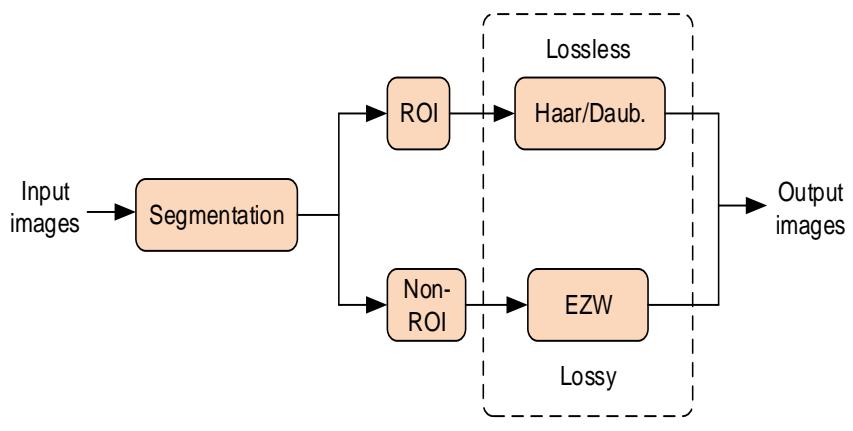

Figure 9. Lossless and lossy compression scheme [31]

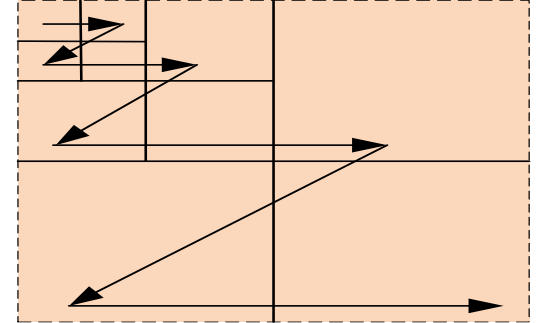

Figure 10. EZW scanning order [1]

Referring to the current research trends regarding medical image compression [1-26], most of the researcher's tendency to conduct a simulation analysis. Furthermore, most of them, explore the performance in terms of PSNR and CR. These because of researchers focusing on the quality of the reconstructed images without thinking the effect of the algorithm development to the hardware performance. Ultimately, algorithm developments for medical image compression frequently explored to achieve better performance in terms of compressed and reconstructed images. More importantly, curvelet transform was the efficient mechanization for medical images compression compared to wavelet transform. Thus, a deep research on curvelet transform need to be explored for future works. Table 1 summarized the previous works based on compression algorithm developments. 
Table 1. Summary of compression algorithm developments

\begin{tabular}{cccc}
\hline References & Wavelet & Curvelet & Evaluation parameters \\
\hline$[1]$ & $/$ & & PSNR \\
{$[13]$} & $/$ & & PSNR \\
{$[15]$} & $/$ & & PSNR and CR \\
{$[27]$} & $/$ & & PSNR and CR \\
{$[28]$} & & $/$ & PSNR and CR \\
{$[29]$} & & $/$ & PSNR and CR \\
{$[30]$} & $/$ & & PSNR \\
{$[31]$} & $/$ & & PSNR \\
\hline
\end{tabular}

\section{ALGORITHM DEVELOPMENT AND HARDWARE-BASED IMPLEMENTATION FOR MEDICAL IMAGE COMPRESSION}

Algorithm development is the nature of image compression system. Indirectly, the simulation-based analysis become the most popular method in order to evaluate the performance of the developed algorithm. The following summarization works are based on algorithm development and also hardware-based implementation for medical image compression. An efficient implementation of medical image compression system using context-based adaptive variable length coding (CAVLC) is described in [32]. The proposed system architecture is targeted for 3-D medical images and has been implemented on Xilinx University Program XUPV5-LX110T Development System. Moreover, user can choose either integer transform (IT) or DWT (Haar filter) to reconfigure the system. Original implementation with a volume $4 \times 4 \times 4$ voxels of CT, MRI and PET images were conducted. Results have shown that using CAVLC, the proposed system can run at a maximum frequency of $152 \mathrm{MHz}$. Moreover, the parallelism design of the proposed architecture has speedup most of the coefficient computations and reduce the usage of internal random-access memory (RAM) and registers.

For implementation approach, shows a variety of design techniques had been used such as pipelinedirect mapping, DA and SA. As reported in [33], FPGA-based implementation using pipeline-direct mapping for medical image compression is proposed. The system architectures utilize Daubechies 4-tap (Daub4) and 6-tap (Daub6) wavelet filters with transposed based computation. Moreover, FPGA would provide efficient performance in terms of computation's speed, area implementation and power consumption. Besides that, user can re-configure the architecture design because FPGA have properties of reconfigurable system. The proposed architecture was implemented using Altera FPGA devices, the cyclone II (EP2C35F672C6) with capacity of 68, 416 logic elements and 250 M4K RAM blocks. Whilst, three parameters have been selected including the area, maximum frequency and power consumption.

Other researcher proposed a method of cascade processing of FPGA-based transformation architectures [13]. The transformation algorithm were computes using DA technique since this technique replace the multipliers which accupy large areas by look up tables (LUTs). All possible coefficient values were stored in the LUT and two input vectors are used to access the LUT content. Moreover, a hybrid method was applied in which a component level IP (CLIP) tool were used to upload the algorithm computation codes with the National Instrument (NI) single-board RIO (sbRIO-9632). Results show, the resolution of 3-D reconstructed image with $N \times N \times N$ points was improved. Furthermore, using DA technique the implementation results proves that the proposed architecture requires less area and low power consumption.

Another study by Iuliana [34] proposed a real-time configurable system to process a medical image using FPGA device. The fact that the medical image contains a complex computation algorithm and thus it is suitable to be implemented on FPGA. In addition, FPGA offers high performance application, flexibility, easy upgradability and low development cost. The proposed model was programmed using Verilog hardware description language (HDL) and an input $400 \times 400$ size of MRI image was stored in an external file. Before the programs being downloaded on the Xilinx virtex-6 FPGA ML605 device, the verilog codes was simulated using Xilinx ISE design suite software.

In order to provide a brief snapshot of existing techniques for medical image compression, Table 2 summarizes the previous works along with the key ideas. It can be observed that various elements and techniques are used in the three components, which are types of compression, implementation and evaluation. In the following works, the cases are used medical modalities such as ultarsound, PET, MRI, CT and X-ray images. From the table, it justifies that more works on algorithm development with the hardware implementation are needed to be carried out for the future works. Furthermore, it is a lot of objective tests was conducted especially in terms of PSNR and CR in order to analyze the performance of the proposed architectures. Thus, it is important to conduct a subjective test, in which the quality of the reconstructed image should be evaluated by the expert in the medical fields. 
Table 2. Summary of Medical Image Compression System

\begin{tabular}{|c|c|c|c|c|c|c|}
\hline \multirow[t]{2}{*}{ References } & \multicolumn{2}{|c|}{ Compression } & \multicolumn{2}{|c|}{ Implementations } & \multicolumn{2}{|c|}{ Evaluations } \\
\hline & Lossy & Lossless & Software & Hardware & Objective & Subjective \\
\hline [13] & & I & VHDL & Spartan-3, FPGAs & I & \\
\hline [33] & & I & VHDL & Cyclone II, FPGAs & / & \\
\hline [34] & & / & Verilog & Virtex-6, FPGAs & I & \\
\hline [35] & & / & MATLAB & & / & \\
\hline [36] & & / & / & & / & \\
\hline [37] & I & / & I & & I & \\
\hline [38] & I & / & VHDL & Zynq-7020, FPGAs & l & \\
\hline [39] & & / & / & & I & \\
\hline [40] & & 1 & 1 & & I & \\
\hline
\end{tabular}

\section{CONCLUSIONS AND FUTURE POTENTIAL RESEARCH}

In conclusion, most of the methodologies on the algorithm development discussed in this review have used the same method; wavelet transform. Eventhough previous works shows a good performance using wavelet transform method, but recently researcher have identified the disadvantage of wavelet transform dealing with medical images. Furthermore, the algorithm development using curvelet transform have become the trends recently for the medical image compression applications and need to be explored more for future works. Moreover, the overall picture of the medical image compression landscape illustrates most of the researchers more focused on algorithm development without having the hardware implementations. In terms of hardware implementation, it has reveals that the complex algorithm computations of medical image processing were suitable to be implemented using FPGA compared to other devices. With the reviews being discussed, this paper opens up extensive research for the algorithm development and implementation in medical compression system. Further study will be done, in which it is expected to have more exploration on curvelet transform algorithm development as well as the hardware-based implementation focusing on medical image compression.

\section{ACKNOWLEDGEMENTS}

The authors would like to thank the Universiti Kuala Lumpur (UniKL) and Majlis Amanah Rakyat (MARA) for funding this research work through Mara Research \& Innovation Grant Scheme (SGPIM).

\section{REFERENCES}

[1] A. Dhivakar, M. G. Ahamad, and D. Ravichandran, "Medical image compression using embedded zerotree wavelet (ezw) coder," in 2016 International Conference System Modeling \& Advancement in Research Trends (SMART), 2016.

[2] A. H. Y. Saad and M. Z. Abdullah, "Real-time implementation of fractal image compression in low cost fpga," in 2016 IEEE International Conference on Imaging Systems and Techniques (IST), 2016, pp. 13-18.

[3] A. Bousselham, O. Bouattane, M. Youssfi, and A. Raihani, "Thermal effect analysis of brain tumor on simulated t1 weighted mri images," in 2018 International Conference on Intelligent Systems and Computer Vision (ISCV), 2018, pp. $1-6$.

[4] A. Ibrahim, J. Thiran, and G. D. Micheli, "Towards ultrasound everywhere: a portable 3d digital back-e-end capable of zone and compound imaging," in IEEE Transactions on Biomedical Circuits and Systems, 2018, pp. 1-14.

[5] M. G. A. D. Ravichandran and A. Dhivakar, "Performance analysis of three-dimensional medical image compression based on discrete wavelet transform," in 22nd International Conference on Virtual System and Multimedia (VSMM), 2016, pp. 1-8.

[6] B. Krill, A. Ahmad, A. Amira, and H. Rabah, "An efficient fpga-based dynamic partial reconfiguration design flow and environment for image and signal processing ip cores," Journal of Signal Processing: Image Communication, vol. 25, no. 5, pp. 377-387, June 2010.

[7] C. Amiot, J. Pescatore, and M. Desvignes, "Curvelet based contrast enhancement in fluoroscopic sequences," in IEEE Transactions on Medical Imaging, 2015, pp. 137 - 147.

[8] P. Sharma, "Dsp in image processing," International Journal of Advanced Research in Computer and Communication Engineering, vol. 4, no. 1, pp. 46-49, Jan. 2015.

[9] N. Prajwal, S. K. Amaresha, and S. S. Yellampalli, "Low power asic implementation of signed and unsigned wallacetree with vedic multiplier using compressors," in 2017 International Conference On Smart Technologies For Smart Nation (SmartTechCon), 2017, pp. 750 - 753.

[10] E. Imam and G. Abdalla, "Design and implementation of discrete cosine transform algorithm on fpga device," in 2016 Conference of Basic Sciences and Engineering Studies (SGCAC), 2016, pp. 13-18.

[11] K. Kalyani, P. P. Omnath, K. Priyadarshani, S. Srinath, and S. Rajaram, "Fpga implementation of fully parallel distributed arithmetic based dct architecture," in 2015 International Conference on Innovations in Information, Embedded and Communication Systems (ICIIECS), 2015, pp. 1-5. 
[12] W. Ayadi, W. Elhamzi, and M. Atri, “A fpga-based implementation of jpeg encoder," in International Image Processing Applications and Systems Conference 2016, 2016.

[13] A. Muharam and A. Ahmad, "Fpga-based architecture of 3-d hwt using distributed arithmetic (da), " in 2014 IEEE Conference on Biomedical Engineering and Sciences, 2014.

[14] C. Kamargaonkar and M. Sharma, "Hybrid medical image compression method using spiht algorithm and haar wavelet transform," in 2016 International Conference on Electrical, Electronics, and Optimization Techniques (ICEEOT), 2016, pp. $897-900$.

[15] U. Patbhaje, R. Kumar, A. Kumar, and H. N. Lee, “A compression system of medical image using wavelet based sparsification and coding," in 2017 4th International Conference on Signal Processing and Integrated Networks (SPIN), 2017.

[16] S. A. Rein, F. H. Fitzek, C. Guhmann, and T. Sikora, "Evaluation of the wavelet image two-line coder: A low complexity scheme for image compression," Signal Processing: Image Communication, vol. 37, pp. 58-74, 2015.

[17] P. Sakthivel and T. K. Devi, "Efficient low power design of 4-tap and 6-tap 2d daubechies wavelet filters using pipelined direct mapping method," Asian Journal of Research in Social Sciences and Humanities, vol. 6, no. 6, pp. 1453-1470, June 2016.

[18] P. Sigh, P. Singh, and R. K. Sharma, "Jpeg image compression based on biorthogonal, coiflets and daubechies wavelet families," International Journal of Computer Applications, vol. 13, pp. 0975-8887, 2011.

[19] M. Nagabushanam, P. C. P. Raj, and S. Ramachandran, "Design and fpga implementation of modified distributive arithmetic based dwt-idwt processor for image compression," in 2011 International Conference on Communications and Signal Processing, 2011, pp. 1-4.

[20] D. U. Shah and C. H. Vithlani, "Efficient implementations of discrete wavelet transforms using fpgas," International Journal of Advances in Engineering \& Technology, vol. 1, pp. 100-111, September 2011.

[21] V. K. Kanth and P. Samundiswary, "Two level 2-d dwt convolution structure for high speed operation using daubehies wavelet," in 2015 IEEE International Conference on Electrical, Computer and Communication Technologies (ICECCT), 2015, pp. 1-5.

[22] H. Al-Marzouqi and G. AlRegib, "Curvelet transform with learning-based tiling," Signal processing: Image communication, vol. 53, pp. 24-39, 2017.

[23] A. Lakshmi, T. Arivoli, and R. Vinupriyadharshini, "Noise and skull removal of brain magnetic resonance image using curvelet transform and mathematical morphology," in 2014 International Conference on Electronics and Communication Systems (ICECS), 2014.

[24] T. Bruyla, A. Munteanu, and P. Schelkens, "Wavelet based volumetric medical image compression," Signal Processing: Image Communication, vol. 31, pp. 112-133, 2015.

[25] E. J. Candes and D. L. Donoho, Curvelets - a surprisingly effective nonadaptive representation for objects with edges, V. U. Press, Ed. Nashville, TN, 2000.

[26] D. Ravichandran, R. Nimmatoori, and M. R. A. Dhivakar, "Performance of wavelet based image compression on medical images for cloud computing," in 2016 3rd International Conference on Computing for Sustainable Global Development (INDIACom), 2016, pp. 297-302.

[27] B. Perumal and M. P. Rajasekaran, "A hybrid discrete wavelet transform with neural network back propagation approach for efficient medical image compression," in 2016 International Conference on Emerging Trends in Engineering, Technology and Science (ICETETS), 2016, pp. 1-5.

[28] M. L. A. Paithane, "Implementing image compression using transform based approach," in 2017 International Conference on Computing Methodologies and Communication (ICCMC), 2017, pp. $834-840$.

[29] P. Anandan and R. S. Sabeenian, "Medical image compression using wrapping based fast discrete curvelet transform and arithmetic coding," Circuits and Systems, vol. 7, pp. 2059-2069, 2016.

[30] V. Yadav, M. Verma, and V. D. Kaushik, "A hybrid image compression technique for medical images," in 2015 International Conference on Computational Intelligence and Communication Networks (CICN), 2015, pp. 222 - 227.

[31] B. V. Reddy, P. B. Reddy, P. S. Kumar, and A. S. Reddy, "Lossless compression of medical images for better diagnosis," in 2016 IEEE 6th International Conference on Advanced Computing (IACC), 2016.

[32] A. Ahmad, A. Amira, M. Guarisco, H. Rabah, and Y. Berviller, "An efficient implementation of medical image compression system using context-based adaptive variable length coding (cavlc)," in IEEE 17th International Conference on Image Processing, 2010, pp. 3773-3776.

[33] A. Ahmad, N. H. Jaafar, and A. Amira, "Fpga-based implementation of 3-d daubechies for medical image compression," in IEEE EMBS International Conference on Biomedical Engineering and Sciences, 2012, pp. 683-688.

[34] I. Chiuchisan, "A new fpga-based real-time configurable system for medical image processing," in The 4th IEEE International Conference on E-Health and Bioengineering - EHB 2013, 2013.

[35] D. Ravichandran, R. Nimmatoori, and A. Dhivakar, "Performance of wavelet based image compression on medical images for cloud computing," in 2016 International Conference on Computing for Sustainable Global Development (INDIACom), 2016, pp. 297-302.

[36] R. Pizzolante, A. Castiglione, B. Carpentieri, and A. D. Santis, "Parallel low-complexity lossless coding of three dimensional medical images," in 2014 International Conference on Network-Based Information Systems, 2014, pp. 91-98.

[37] R. R. S. Tomar and R. R. S. Tomar, "Lossless image compression using differential pulse code modulation and iapplication," in 2015 Fifth International Conference on Communication Systems and Network Technologies, 2015, pp. 543-545. 
[38] P. Govindan, B. Wang, P. Ravi, and J. Saniie, "Hardware and software architectures for computationally efficient three dimensional ultrasonic data compression," IET Circuits, Devices \& Systems, vol. 10, no. 1, pp. 54 - 61, 2016.

[39] S. S. Parikh, D. Ruiz, H. Kalva, G. Fernandez-Escribano, and V. Adzic, "High bit-depth medical image compression with hevc," IEEE Journal of Biomedical and Health Informatics, vol. 22, no. 2, pp. 552-560, 2018.

[40] F. Sepehrband, M. Mortazavi, and S. Ghorshi, "Efficient dpcm predictor for hardware implementation of lossless medical brain ct image compression," in The International Conference on Signals and Electronic Systems, 2010, pp. $123-126$.

[41] S. Sani, "A wavelet based solar radiation prediction in Nigeria using adaptive neuro-fuzzy approach," Indonesian Journal of Electrical Engineering and Computer Sciences, vol. 12, no 3, December 2018.

[42] H. L. Man and Y. S. Soo, "Modified Pixels Based Fast Median Filter in Impulse Noise Environments," Indonesian Journal of Electrical Engineering and Computer Sciences, vol. 9, no 3, March 2018.

\section{BIOGRAPHIES OF AUTHORS}
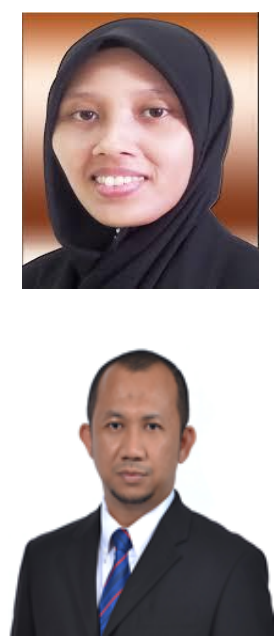

Noor Huda Ja'afar received her B. Eng and M. Eng in Electrical Engineering from Universiti Tun Hussein Onn Malaysia (UTHM) in 2010 and 2013, respectively. She worked as a lecturer at Instrumentation and Control Engineering Section, Universiti Kuala Lumpur Malaysian Institute of Industrial Technology. Currently, she is a Ph.D candidate in the Electrical Engineering, UTHM on the efficient reconfigurable architectures of curvelet transform for $3 \mathrm{D}$ medical image compression. Her research interest includes 3D transform, medical imaging and reconfigurable computing.

Afandi Ahmad has recently be appointed as Deputy Vice Chancellor of student's affair and alumni in Universiti Tun Hussein Onn Malaysia (UTHM). Currently, he is a senior lecturer at UTHM under Faculty of Electrical and Electronic Engineering. He received his Ph.D. In Electronic and Computer Engineering at Brunel University, London in 2010. He has been awarded a number of grants from government and has published over 130 publications during his career to date. He is a member of IEEE, IAENG, IET and BEM. His research interests include: embedded systems, reconfigurable computing, image processing, medical applications. 